\title{
What new immunotherapeutic techniques are currently being investigated for the treatment of
} melanoma?

\section{"Inevitably, when looking for the next boost to survival curves, there is a tendency to focus on the next 'wonder molecule', but there are other equally important approaches to heed."}

\section{KEYWORDS: adoptive cell $=$ IL-2 - immunotherapy $\approx$ melanoma $\approx$ oncolytic virus}

We've waited decades for a treatment that actually helps patients with metastatic melanoma, and then, like buses, two come along at once. Both vemurafenib and ipilimumab have, in Phase III trials, demonstrated improved overall survival in patients with metastatic melanoma [1,2]. Two comforting fillips for the field, but sadly it is not time for anyone with an interest in melanoma to pack up and go home just yet; each agent produced only modest benefits. What then is coming next? Are there more buses on the way, to pick up those who could not cram into the first two?

The discovery and purification of IL-2, and its early use in melanoma, have tied research into immunotherapies inextricably to melanoma. Although IL-2 is still hailed by some proponents as a viable and effective treatment, it remains expensive and has marked adverse effects; there is certainly consensus that its use should be restricted to expert centers only [3]. Understanding of the interaction of IL-2 and its cell surface receptors has led to the development of a 'super-IL-2' [4]. IL-2, bound to its cell surface receptor, normally needs the contribution of CD25 to optimize its function; super-IL2 has, through the use of an evolutionary approach in yeast, circumvented the requirement for $\mathrm{CD} 25$, thus enhancing its effects. The modified cytokine has improved efficacy and reduced toxicity in preclinical models, although its effect in humans remains to be seen. Conventional IL-2 has, however, continued to be a vital component of Steven Rosenberg's work at the National Cancer Institute (MD, USA), where he has been developing adoptive cell therapy (ACT) for over 20 years. Unquestionably, the response rates at Bethesda are impressive; in the most recently reported protocol (which includes intensive preconditioning with ablative chemotherapy and radiotherapy), 10/25 patients responded completely, and historically most ACT patients who respond that well have not relapsed after many years of follow-up [5]. These were preselected and fit patients, but to cure metastatic disease remains hugely impressive in the context of melanoma. The concern is that the approach has not been adapted by many centers because the treatment is technically, economically and physically very challenging.

A recent development, which may allow the use of ACT to expand, are T cells engineered to express chimeric antigen receptors (CAR-T). The original ACT protocol requires surgical excision of tumors that are then used to isolate and expand tumor-infiltrating T cells; not a trivial undertaking. CAR-T cells can instead be prepared from peripheral blood, their tumor specificity conferred by a lentiviral vector encoding an extracellular antibody fragment and costimulatory intracellular domains that can serve to activate the cells in lieu of cognate antigen recognition by the native T-cell receptor [6]. CAR-T cells are not subject to MHC restriction and therefore the difficulties of matching HLA type with available epitopes are neatly sidestepped. This approach, while not yet perfected, has shown exciting promise in other tumor types and could be an important evolution of Rosenberg's ACT.

Treatments such as radiotherapy and chemotherapy are now known to drive the production of adaptive antitumor responses, albeit generally to an extent that they can be demonstrated in the laboratory, rather than producing clinical therapeutic immunity. In this context, it is clear that generating specific $T$ cells is not enough - tumor-driven immunosuppresion must also be overcome. Antibodies against CD25 (chiefly daclizumab) are being tested in several clinical trials at present for their ability to deplete regulatory $\mathrm{T}$ cells, and offer several combinatorial possibilities [7]. Focusing on a single element of
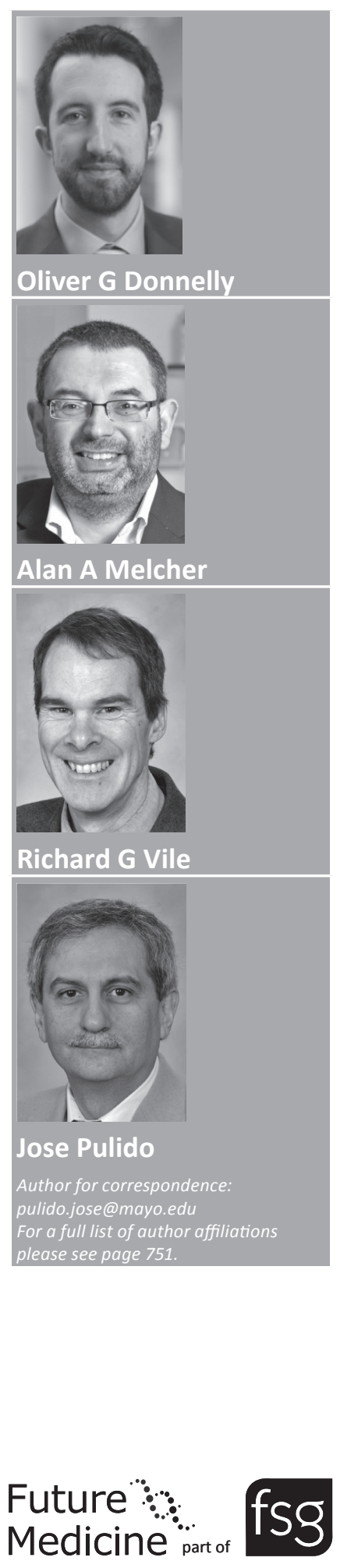
the immune response is akin to trying to play chess using only the rooks; the occasional win is possible, but it is probably not the ideal strategy.

In addition to its efficacy, ipilimumab has demonstrated considerable toxicity, and improvements in tolerability may emerge if the systemic dysregulatory effects of CTLA-4 blockade can be better directed, perhaps by generating local areas of tumor antigen release and danger signals using chemotherapy or radiotherapy. There are already case reports of synergy between ipilimumab and stereotactic radiotherapy [8], and several combination trials are listed on the clinicaltrials.gov website [101].

\section{"In the application of chemotherapy and molecular targeting, there is considerable focus on personalizing medicine, but it seems somewhat lacking within the study of immunotherapy."}

So many conventional therapies are being discovered to have immunological effects that it is almost becoming the assumed norm. Accordingly, a preclinical study with vemurafenib has revealed its ability to increase antigen expression in melanoma lines bearing $B R A F$ mutations, and unlike MEK inhibitors that act further downstream, it does not impair T-cell function [9]. There is an obvious potential benefit in its combination with CTLA-4 blockade, both mechanistically and pragmatically. Vemurafenib has higher response rates than ipilimumab, but ipilimumab seems to generate more durable responses. Therefore, the combination may have strong additive benefits and a good chance of significant synergy, and is due to be tested clinically [102]. As an ancillary issue it will be fascinating to see whether CTLA-4 blockade has any impact on the established proclivity of vemurafenib to cause cutaneous keratocanthomas/squamous cell carcinomas.

An emerging target is the programmed cell death 1 receptor/ligand (PD-1/PD-L1) pairing. Much like CTLA-4, these moieties serve to curb immune responses by interfering with the immune synapse, and one of the several anti-PD1 antibodies being developed (MDX1106) has shown promise in early trials [10]. PD-L1 may be a more interesting target as it is also known to be overexpressed on melanoma cells, thus offering a combination of immune deregulation as well as direct labeling of melanoma cells for antibodydependent cellular cytotoxicity [11]. Several other elements of the immune synapse are also being targeted.
Oncolytic viruses, initially explored for their ability to directly lyse cancer cells are increasingly thought to rely on stimulating host antitumor immunity, and several of these viruses have been armed with immunologically active molecules, particularly GM-CSF. The current poster child for the field, OncoVex (talimogene laherparepvec; Amgen Inc, CA, USA), is a modified type I herpes virus that encodes GM-CSF [12]. Results from a Phase II trial in melanoma showed sufficient promise to prompt Amgen to commit up to US $\$ 1$ billion to further development of the virus and results from the Phase III melanoma trial are keenly awaited, along with results from other viruses in earlier-stage testing.

Inevitably, when looking for the next boost to survival curves, there is a tendency to focus on the next 'wonder molecule', but there are other equally important approaches to heed. Hematologists dramatically improved the outlook for children with acute myeloid leukemia, not because of new drug targets but instead by methodical refinement of supportive care and dose intensity [13]. In a broadly similar vein, our colleague Svetomir Markovic has demonstrated the highly dynamic nature of immune responses and the importance of immune biorhythms. Following up on intriguing retrospective data, Markovic's team is exploring the benefits and practicability of individualized treatment synchronization, better to harness the immune priming potential of various chemotherapeutics [14].

In the application of chemotherapy and molecular targeting, there is considerable focus on personalizing medicine, but it seems somewhat lacking within the study of immunotherapy. We know too little about what separates those who benefit from ipilimumab, or any of the other treatments described above, from those who do not. It is becoming clear that developing autoimmunity is a reasonable predictor of successful antitumor immunity but it is imperative to find pretreatment predictive markers in order to reduce toxicity and improve cost-effectiveness. The lesson from the troubled development of Iressa ${ }^{\circledR}$ must be that response rates are improved by selecting the right patients (or perhaps more accurately excluding the wrong patients). We must access patients earlier in the course of their disease. Many in the field are of the view that successful immunotherapy is much more likely to be achieved in good performance status patients, and the adjuvant setting is particularly appealing. The traditional Phase I-III model is appropriate for many oncological approaches but 
probably does not serve immunotherapies well. Successful immunotherapy will be complicated. Multiple facets of the host immune response will need to be assessed and manipulated in an ad personam fashion, out of keeping with current treatment approaches. Tumor immune suppression must also be overcome. Such complexity and individualization does not readily lend itself to therapeutic protocols that are likely to be considered favorably by review boards and funders, and this organizational hurdle could yet be the major obstacle for immunotherapy.

Newly approved treatments have improved the outlook for metastatic melanoma considerably, but incompletely. Immunotherapeutic

\section{References}

1 Chapman PB, Hauschild A, Robert C et al. Improved survival with vemurafenib in melanoma with BRAF V600E mutation. N. Engl. J. Med. 364, 2507-2516 (2011).

2 Hodi FS, O’Day SJ, McDermott DF et al. Improved survival with ipilimumab in patients with metastatic melanoma. N. Engl. J. Med. 363(8), 711-723 (2010).

3 Rosenberg SA. Raising the bar: the curative potential of human cancer immunotherapy. Sci. Transl. Med. 4(127), 127ps8 (2012).

4 Levin AM, Bates DL, Ring AM et al. Exploiting a natural conformational switch to engineer an interleukin-2 'superkine'. Nature 484, 529-533 (2012)

5 Rosenberg SA, Yang JC, Sherry RM et al. Durable complete responses in heavily pretreated patients with metastatic melanoma using T-cell transfer immunotherapy. Clin. Cancer Res. 17, 4550-4557 (2011).

6 Porter DL, Levine BL, Kalos M, Bagg A, June $\mathrm{CH}$. Chimeric antigen receptor-modified $\mathrm{T}$ cells in chronic lymphoid leukemia. N. Engl. J. Med. 365, 725-733 (2011).

7 Rech AJ, Vonderheide RH. Clinical use of anti-CD25 antibody daclizumab to enhance immune responses to tumor antigen vaccination by targeting regulatory $\mathrm{T}$ cells. Ann. NY Acad. Sci. 1174, 99-106 (2009).

8 Postow MA, Callahan MK, Barker CA et al. Immunologic correlates of the abscopal effect

approaches show promise, and despite significant challenges still to be overcome, there seems to be scope for optimism.

\section{Financial \& competing interests disclosure}

OG Donnelly is supported by grants from the Medical Research Council (UK) and the Ellison-Cliffe Travelling Fellowship from the Royal Society of Medicine, London (UK). The authors have no other relevant affiliations or financial involvement with any organization or entity with a financial interest in or financial conflict with the subject matter or materials discussed in the manuscript apart from those disclosed.

No writing assistance was utilized in the production of this manuscript.

in a patient with melanoma. N. Engl. J. Med. 366, 925-931 (2012).

9 Boni A, Cogdill AP, Dang P et al. Selective BRAFV600E inhibition enhances T-cell recognition of Melanoma without affecting lymphocyte function. Cancer Res. 70, 5213-5219 (2010).

10 Brahmer JR, Drake CG, Wollner I et al. Phase I study of single-agent antiprogrammed death-1 (MDX-1106) in refractory solid tumors: safety, clinical activity, pharmacodynamics, and immunologic correlates. J. Clin. Oncol. 28, 3167-33175 (2010)

11 Garber K. Beyond ipilimumab: new approaches target the immunological synapse. J. Natl Cancer Inst. 103(14), 1079-1082 (2011).

12 Senzer NN, Kaufman HL, Amatruda T et al. Phase II clinical trial of a granulocytemacrophage colony-stimulating factorencoding, second-generation oncolytic herpesvirus in patients with unresectable metastatic melanoma. J. Clin. Oncol. 27, 5763-5771 (2009).

13 Gibson BE, Wheatley K, Hann IM et al. Treatment strategy and long-term results in paediatric patients treated in consecutive UK AML trials. Leukemia 19, 2130-2138 (2005).

14 Holtan SG, Dronca RS, Nevala WK et al. The dynamic human immune response to cancer: it might just be rocket science. Immunotherapy 3, 1021-1024 (2011).

\section{Websites}

101 ClinicalTrials.gov. www.clinicaltrials.gov

$102 \mathrm{Ph} \mathrm{I/II} \mathrm{Ipilimumab} \mathrm{Vemurafenib} \mathrm{Combo.}$ www.clinicaltrials.gov/ct2/show/NCT01400 451?term $=$ NCT01400451\&rank=1

\section{Affiliations}

- Oliver G Donnelly

Leeds Institute of Molecular Medicine, University of Leeds, Leeds, UK and

Mayo Clinic, 200 First St SW, Rochester, MN 55902, USA

- Alan A Melcher

Leeds Institute of Molecular Medicine, University of Leeds, Leeds, UK

- Richard G Vile

Leeds Institute of Molecular Medicine, University of Leeds, Leeds, UK and

Mayo Clinic, 200 First St SW, Rochester, MN 55902, USA

- Jose Pulido Mayo Clinic, 200 First St SW, Rochester, MN 55902, USA 